\title{
LA PUTXERAMOBIL, EL DÍA DE GANGAS
}

\section{Laurita Siles Ceballos}

Universidad del País Vasco / Euskal Herriko Unibertsitatea. Dpto. Arte y Tecnología. Doctoranda

\section{Resumen}

La Putxeramobil es una putxera sobre una bicicleta. La Putxeramobil nació con el objetivo de devolver el movimiento al proceso de preparación y cocción de las alubias a partir de la simple y ecológica tracción humana del pedaleo de una bicicleta. Las alubias se cocinan al ritmo del pedaleo mientras los bertsolaris dinamizan su preparado; se continúa con una suculenta comida popular y sigue un recital de sobremesa. A la vez que se reivindican elementos de la cultura popular local desde la práctica artística en un contexto festivo y participativo, se potencia el euskera en la zona de Enkarterri, invitando a los transeúntes a que se animen a participar en este recorrido a cambio de un suculento plato de alubias. Tanto en la producción como la ejecución de este proyecto han interferido un gran número de personas y entes locales, y se han generado abundantes interrelaciones humanas desde la experimentación colaborativa a partir de estrategias artísticas y sociales.

\section{Palabras clave: PUTXERA; BICICLETA; BERTSOLARISMO; ARTE; ENKAR-} TERRI (BIZKAIA, COMARCA)

\section{THE PUTXERAMOBIL, THE DAY OF BARGAINS}

\section{Abstract}

The Putxeramobil is a putxera on a bicycle. The Putxeramobil was designed to bring back movement to the preparation and cooking process of beans through the simple and ecological human-powered of pedaling a bicycle. Beans are cooked to the beat of pedals while bertsolaris infuse their energy to the process. When the succulent popular meal is tasted, a table-talk recital is performed. From the artistic practice, local popular and cultural elements are claimed in a participatory and festive context while the Euskera language is promoted in Enkarterri area and passers-by are invited to join to the route in exchange for a plate of beans. A high number of persons and local organizations have been involved both in production and execution of the project. From artistic and social strategies lots of human relations take place from collaborative experimentation.

Key words: PUTXERA; BIKES; BERTSOLARISM; ART; ENKARTERRI (BIZKAIAN DISTRICT)

Siles Ceballos, Laurita. 2017 . "La Putxeramobil, el día de gangas". AusArt 5 (1): 229-241 DOI: 10.1387/ausart.17111

\section{AUSART}




\section{INTRODUCCIÓN}

La Putxeramobil es una putxera sobre una bicicleta. La idea de este singular velocípedo surgió a raíz de conocer a Joseba Edesa y Mikel, en enero de 2013; ambos, por aquel entonces, vivían en el apeadero de la estación de tren de la Feve Arla-Berrón, en una casa situada en la muga entre Bizkaia y Burgos; donde cada día siguen parando dos trenes de pasajeros dirección Burgos-Bilbao-Burgos, junto a otros tantos trenes de mercancía, que no realizan parada alguna, solo pasan. El apeadero de Arla-Berrón, para la mayoría de las personas que lo conocen y transitan, es un lugar de itinerarios individuales. Como describió Marc Augé, es un no lugar, un lugar que carece de la configuración del espacio, definido casi exclusivamente por el pasar de individuos (Augé 1998). Un lugar de tránsito para los viajeros, pero para estos dos amigos Arla-Berrón se convirtió por algún tiempo en un hogar. Un curioso 'no lugar' convertido en 'lugar'.

Además, hay que decir que Joseba y Mikel desarrollan su vida social en la zona de Enkarterri, en Bizkaia, y acostumbraban a cocinar una putxera de alubias en fechas señaladas o con motivo de reunión junto a familiares y amigos.

La putxera es un icono gastronómico y festivo principalmente de la zona Enkarterri; un utensilio fruto de la sabiduría popular, procedente del trayecto ferroviario de Bilbao-La Robla (León), y la estación Arla-Berrón forma parte de este trayecto. La putxera surgió sobre el siglo XIX, a raíz del ingenio de los maquinistas que idearon una particular olla para cocinar que obtenía la fuente de calor del carbón del tren que se almacenaba en la parte baja del utensilio, confiriéndole un toque suculento al tradicional cocido de alubias; de esta manera conseguían comer un plato caliente en los crudos inviernos que vivieron.

Hoy en día la putxera está presente en las fiestas populares de la zona, pero el proceso de su elaboración ya no es móvil. La Putxeramobil nació con el objetivo de devolver el movimiento al proceso de preparación y cocción de las alubias; a partir de la simple y ecológica tracción humana del pedaleo de una bicicleta. Así que, una vez construida la Putxeramobil, decidimos sacarla a la calle. Para ello, realizamos tres diferentes acciones participativas en las localidades de Zalla, Balmaseda y Bilbao, lugares por donde transita, a día de hoy, el emblemático tren de La Robla'1. 
Las acciones se llevaron a cabo durante tres fechas concretas:

- En Zalla, el Día de Gangas, que se celebra el primer lunes de octubre y es una feria tradicional de productos locales (7 de octubre de 2013).

- En Balmaseda, el día del Concurso Mundial de Putxeras (celebrado anualmente el 23 de octubre), festividad de San Severino.

- En Bilbao, el días de Santo Tomás (21 de diciembre). Una jornada en la que reina el ambiente rural y marca el inicio de la Navidad. Se celebra en multitud de municipios de la geografía vasca, entre ellos, Bilbao.

En cada uno de estos actos se realizó un recorrido (de 11:00 de la mañana a 15:00 de la tarde aproximadamente), por algunas de las emblemáticas calles del municipio escogido, con la idea de ir realizando un peculiar bertso-poteo ${ }^{2}$. Se cocinaron las alubias al ritmo del pedaleo, con la dinamización por parte de dos o tres bertsolaris diferentes en cada ocasión, para continuar con una suculenta comida popular, seguida de un recital de sobremesa, comúnmente conocido como bertso-bazkari ${ }^{3}$.

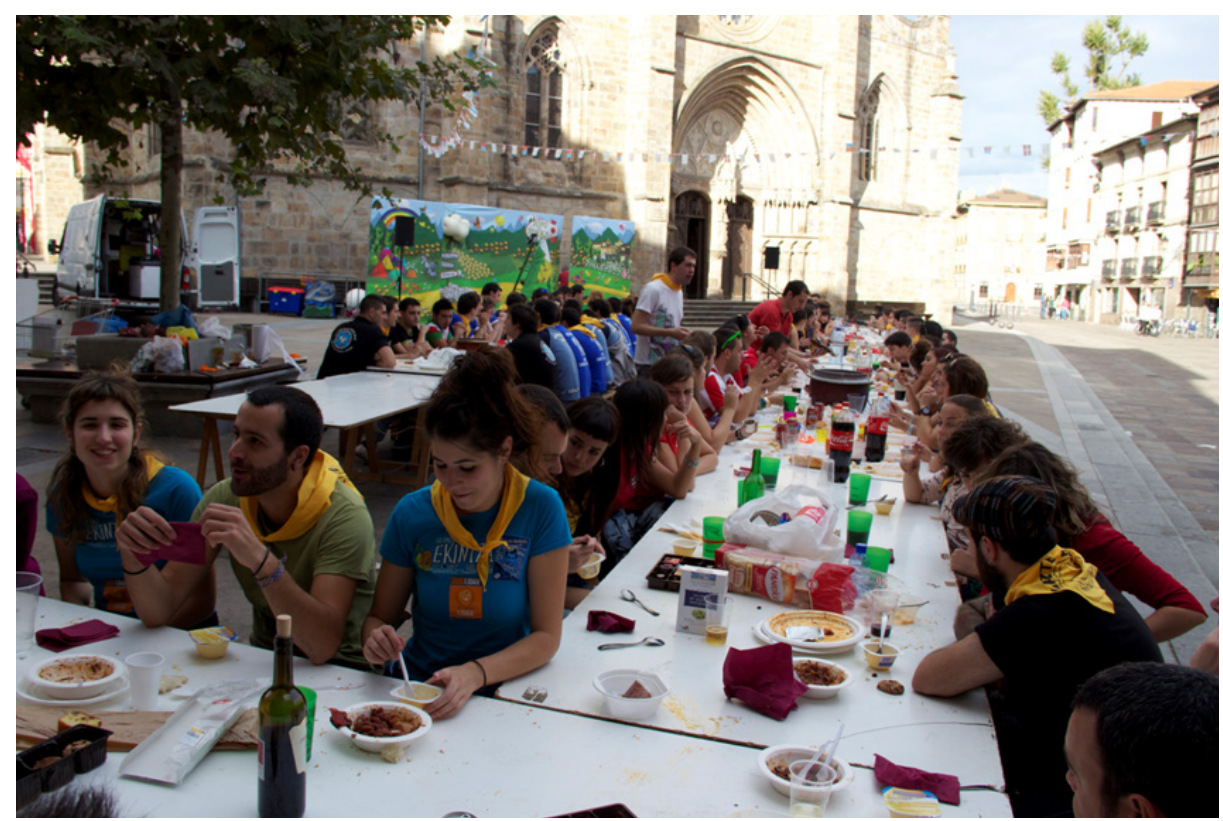

Figura 01: Comida de la Putxeramobil celebrada en Balmaseda, el día de San Severino. Concurso Mundial de Putxeras, 2013. 
Tanto en la producción como la ejecución de este proyecto han interferido un gran número de personas y entes locales. Se generaron así - sin ser apenas conscientes hasta última instancia- abundantes interrelaciones humanas desde la experimentación colaborativa a partir de estrategias artísticas y sociales. Destacó especialmente la acción inaugural de la Putxeramobil realizada en Zalla, el Día de Gangas que a continuación analizaremos con detalle.

\section{LA PUTXERAMOBIL EL DÍA DE GANGAS}

El Día de Gangas es una fecha señalada anualmente en el almanaque de los habitantes de este pueblo. Este es el lunes siguiente al primer domingo de octubre, que coincide con el último día de las fiestas locales. Este día es tan importante para los habitantes de Zalla como para los vecinos de los pueblos limítrofes, ya que se celebra la feria más antigua de la comarca, junto con un concurso agrícola. Allí las baserritarras (hortelanos y hortelanas) muestran los mejores productos de su cosecha: pan, miel, queso, frutas, hortalizas, txakoli, etc. También tiene cabida una exposición de maquinaria agrícola, danzas, artesanía, etc. Hay igualmente exposiciones monográficas del sector primario, muestra de ganado local: ovino, bovino, vacuno y caballar.

Lo interesante de la acción de la Putxeramobil el Día de Gangas estuvo en la dinamización colaborativa junto a diferentes entes del municipio. Se contó con la participación de la escuela de bertsolarimos de Zalla Zallako Bertsolari Eskola, y la colaboración y producción de la comisión de fiestas de Zalla Zallako Jai Batzordea y la asamblea de jóvenes de Zalla, Zallako Gazte Asanblada.

Antes de continuar debo aclarar cuál es la actividad de cada uno de estos colectivos:

- La escuela de bertsolarismo de Zalla es un centro de formación y aprendizaje del cante de bertsos. Zallako Bertsolari Eskola fue creada en 2001 y desde entonces ha funcionando en Enkarterri como una herramienta importante para fomentar el euskera. Además, son los encargados de organizar una gran cantidad de eventos que se articulan a lo largo del año en su comarca, como actuaciones en diferentes celebraciones, funerales u homenajes que conciernen al pueblo 
donde viven. También organizan bertso jaialdia (festivales de bertsolarimo), bertso bazkari (comida con sobremesa de bertsolarismo), etc., donde suelen invitar a bertsolaris de otras comarcas.

- Las comisión de fiestas de Zalla -Zallako Jai Batzordea- está formada por todo tipo de personas representantes de comparsas, lonjas, asociaciones, clubs, etc., que quieran participar y colaborar en la organización de las fiestas de su pueblo. Estas se organizan mediante reuniones asamblearias en los meses previos a la fecha de la celebración de las fiestas. Tienen la finalidad de organizar la partida presupuestaria cedida por el Ayuntamiento para el alquiler de escenarios, sillas, mesas, la contratación de grupos de música, bertsolaris, etc., así como todo lo necesario para completar la programación festiva.

- La asamblea de jóvenes de Zalla -Zallako Gazte Asanblada- se creó en el año 2006 con el objetivo de promover la cultura y el ocio participativo entre los jóvenes del pueblo, mediante un sistema asambleario. Los principales objetivos de estos jóvenes como grupo pueden ser tan amplios como la lista de los problemas relacionados con la juventud de su entorno más cercano: la creación de una cultura y un ocio alternativos, el derecho a luchar por la vivienda, denunciar la precariedad, la situación de los derechos lingüísticos, la soberanía alimentaría, etc.

Cada año la Zallako Gazte Asanblada durante la celebración de las fiestas locales dispone de una txosna ${ }^{4}$; y participan de igual forma como integrantes de la comisión de fiestas, organizando actividades de diferente índole, como el concurso de pintxos o la bertso-bazkari. Esta última iniciativa, desde hace unos años, se celebra el Día de Gangas. Es una propuesta intrépida, teniendo en cuenta la escasez del uso del euskera en las Enkarterri, tanto en su historia más reciente, como en la actualidad 5 . 


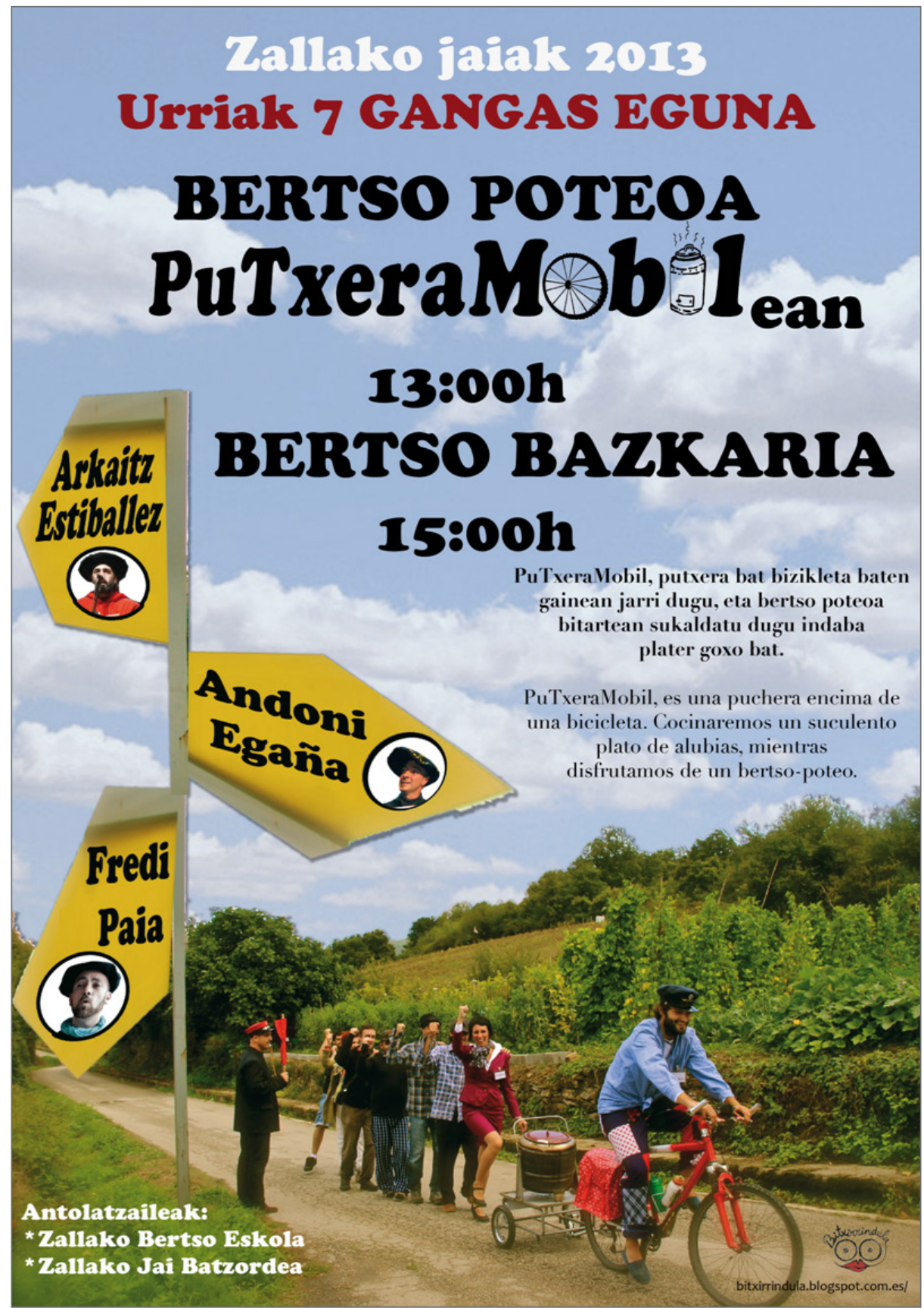


Joseba y Mikel son euskaltzaleak ${ }^{6}$ y amantes del bertsolarismo. Ambos, por aquel entonces, formaban parte activa de la Gazte Asanblada de Zalla, donde desarrollaban una ingente cantidad de actividades en torno a los temas que les conciernen -como la soberanía alimentaria, el derecho a techo, el fracking o el uso y promoción del euskera-, ya que para ambos este municipio es el lugar donde han crecido y donde residen sus familiares y amigos. De tal modo que, de una manera totalmente natural y progresiva, tras nuestras primeras conversaciones, al conocer nuestros gustos y preocupaciones, la Putxeramobil se materializó como una convulsión plena de sinergia y comunicación, claves del trabajo en equipo, y el lugar escogido para su estreno, sin lugar a dudas, debía ser Zalla.

De tal modo que la sesión de bertsos de aquel año 2013, el Día de Gangas en las fiestas de Zalla, además de formar parte de la sobremesa de la comida, se amplió con el bertso-poteo previo, junto al proceso de cocinado. Tal vez por primera vez, el cocinero - de una putxera de alubias - no faltaría en el itinerario del poteo previo a la hora de almorzar. Gracias a la Putxeramobil, este personaje, además de estar presente, formó parte integral del evento e incluso fue protagonista de más de un bertso:

Andoni Egaña:

Bueno putxera ez al da hobe, babarrunak ikusteko.

Egurra hemen alperrik denik, inork ez du ba usteko.

Makinista honek egur zati bat, Jarri gurpilen menpeko

Hemen egurrik gehien behar da, bizikletari eusteko ${ }^{7}$.

Fredi Paia:

Eta oraindino hor Daukagu putxera.

La Roblako trenetik Zallako aldera

Ai makinista jauna Frediren galdera:

Noiz Joan behar dugu?

Hemen bazkaltzera ${ }^{8}$. 
Fredi Paia:

Eguraldia ederra dala,

jaungoikoari eskerrak.

ta euskaldunak sabelaz betez,

sortzen dituen plazerrak

indaba goxoz beteraragu,

bi bertsolari alperrak

ze katalina goxoak

eta zelako pinoi ederrak ${ }^{9}$.

Andoni Egaña:

Platerak ere onak zirela, aitortu egin beharko.

Sukaldaria lehen ibiltzen zen,

poteontatik kanpo.

Gaurkoan gure taldeko gendun, txirrindaba bat zuelako.

Merezi du ta txalo handi bat

sukaldariarentzako ${ }^{10}$.

Fredi Paia:

Txirrindulari sukaldariak, gure platerrak betea.

Izugarria da Euskal Herrian, gastronomian artea

Kasualidadez ni indabetan, aditua izatea

ta ze goxoak gure indabei, E.P.O.ak emandako tokea ${ }^{11}$.

Los bertsolaris invitados para tal evento fueron Andoni Egaña, Fredi Paia y Arkaitz Estiballez, pero este último no pudo asistir, así que la sesión de bertsos fue encaminada entre Fredi y Egaña. Son dos bertsolaris, podría decirse, de alta categoría, ya que en sus espaldas llevan una trayectoria de gran valor en el mundo del bertsolarismo. Andoni Egaña (Zarauz, 1961) ha ganado el Campeonato Nacional de Bertsolaris - Bertsolari Txapelketa Nagusia-, máxima competición de la disciplina, en cuatro ocasiones, y ostenta de este modo el récord de victorias en dicha competición. Además, es una figura muy impor- 
tante del bertsolarismo, no solo por sus logros en campeonatos, sino porque es también uno de los principales teóricos del bertsolarismo moderno. Por otro lado, veinte años más joven, Fredi Paia (Algorta, 1981) ha sido el primer bertsolari de familia de habla no euskalduna que ha llegado a la final del Campeonato Nacional de Bertsolaris en más de una ocasión. También ha sido vencedor del Campeonato de Bizkaia de Bertsolaris en 2014 y, además de improvisar, ha trabajado en otros ámbitos, como en educación y cultura o colaborando en televisión, radio y prensa euskalduna.

El caché o cotización para una actuación en público de los bertsolaris Fredi Paia y Andoni Egaña es de las más elevadas. De tal modo que la colaboración presupuestaria de la comisión de fiestas de Zalla fue clave para la ejecución del mismo, así como la ayuda y predisposición en la organización y cuidado de la acción por parte de la Zallako Bertso Eskola y la Gazte Asanblada. Además, esta última, se encargó de los gastos de todos los ingredientes necesarios para cocinar la putxera (alubias, costilla de cerdo, tocino, chorizos, cebolla, morcillas, pimiento choricero, tomate, puerro, bebida, pan, etc.).

\section{CONCLUSIONES}

En este sentido, es muy interesante señalar la articulación de cómo pueden ser incorporadas las «tecnologías blandas» en los procesos artísticos —según apunta la artista e investigadora Saioa Olmo junto a cooperativa ColaBoraBora $^{12}$ - . A partir de la adecuación de los espacios previos de actuación de los tres promotores de la Putxeramobil, articularon un proyecto que se funde entre lo artístico y lo vital, es decir: por un lado, estarían Joseba y Mikel, desde el campo social y cultural local; por otro, estaría el bagaje creativo desarrollado por la autora de este artículo, en propuestas artísticas anteriores como son la Txalamobili13, la Txalabilbote ${ }^{14}$ o la Bicine ${ }^{15}$. De esta forma se experimentan otras posibilidades de intercambio diferentes a las hegemónicas del sistema imperante en la producción de un proyecto de arte (Bourriaud 1998 citado en Blanco et al. 2011, 432). Esto es así porque, por ejemplo, la Putxeramobil fue un proyecto de arte pensado desde el pueblo y para el pueblo, y gracias a la colaboración del mismo este fue posible, lo que enfatiza la indispensable labor que tiene arte en la educación de la ciudadaní. 


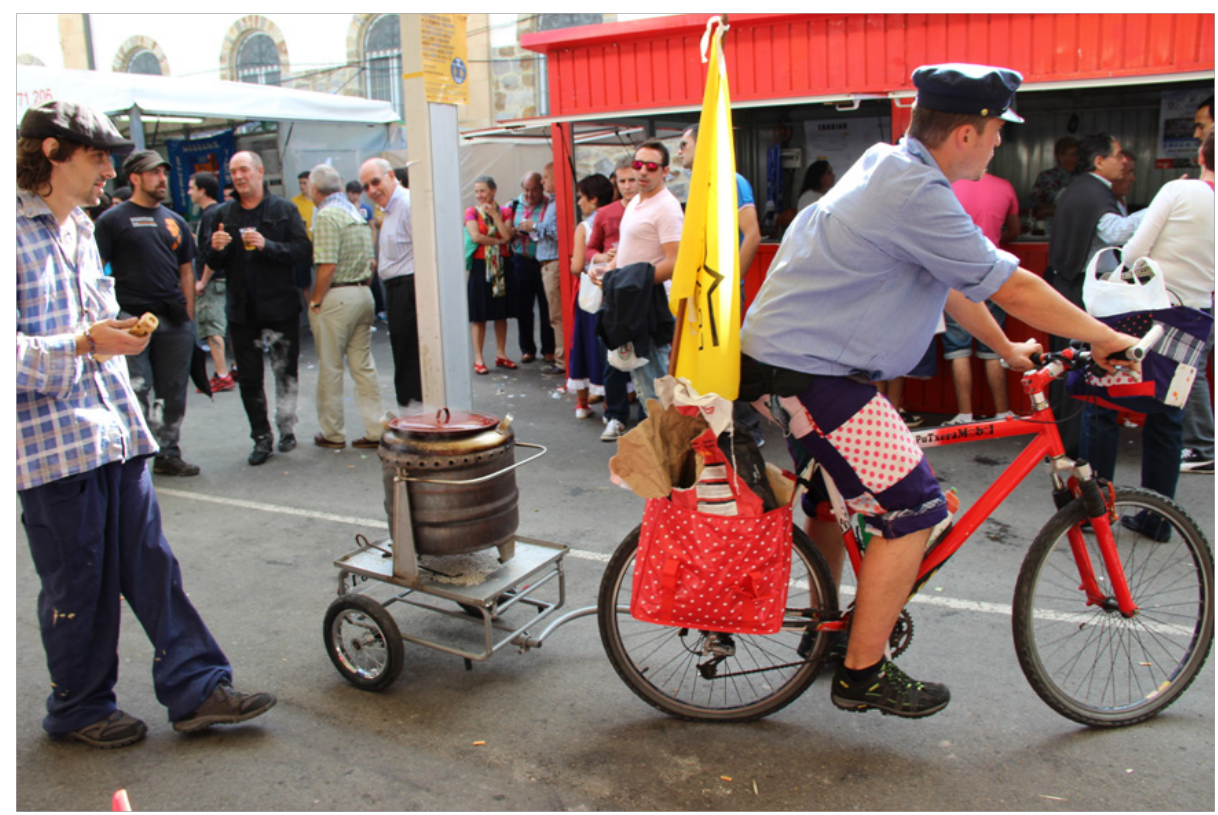

Figura 03: La Putxeramobil. Gangas Eguna, Zalla. 2013.

La Putxeramobil del Día de Gangas en Zalla tuvo una calurosa acogida por el público transeúnte; jóvenes y mayores se acercaron con curiosidad, asombro y alegría. La natural predisposición a la interacción multisensorial estaba latente en un entorno propiciado para la fiesta y el regocijo, según señalaba el calendario local -esto es importante ya que hay que tener en cuenta que la Putxeramobil exige una actitud activa a los espectadores- Se estimulan otros canales expresivos más allá de los métodos convencionales que podemos encontrar tradicionalmente en un museo o galería, donde el visitante solo puede mirar (Lía Bang 2013). Se precisaba que el espectador formara parte activa de la acción: beber y comer, disfrutar de la escucha de los bertsos, caminar tras la Putxeramobil, etc., eran actos imprescindibles para poder experimentar estéticamente la obra junto al encuentro creativo consigo mismo. En este sentido, esta adecuación estimulante, generada desde la práctica artística a partir de la implicación ciudadana, puede encontrar su semejanza en la manera de operar que podemos experimentar a la hora de formar parte de un acto folklórico, es decir, entender un folklore como vivencia. Esto que parece invisible en el presente frente a la visibilidad del folklorismo, entendido este último como vivencia de una vivencia (Díaz Viana 2013, 119; Martí i Pérez 1996). 
De hecho, en relación a esta idea, la acogida del proyecto superó nuestras expectativas, hasta el punto de que fue adoptado por la comunidad de Zalla como una nueva tradición. Desde entonces, la Putxeramobil ha salido a la calle cada año el Día de Gangas. Se genera así una tradición — propia y local- (Hobsbawm \& Ranger 2002) a partir de un proyecto artístico. Todo esto permite reflexionar sobre la dicotomía entre lo culto y lo popular, la vanguardia y la tradición, lo rural y lo urbano, etc. Oposiciones binarias estas, enfrentadas donde los actos festivos locales se nutren del privilegio social del arte. Se reivindican elementos de la cultura popular local, desde la práctica artística, en un contexto local festivo y participativo; a la par que se potencia el euskera en la zona de Enkarterri. Se invita a los transeúntes a que se animen a participar en este recorrido a cambio de un suculento plato de alubias cocinadas en la Putxeramobil. Todo ello bajo el impulso de fomentar la bicicleta como medio de transporte sostenible, contribuyendo a restaurar la vitalidad ambiental y cultural, y devolviendo un profundo significado a la experiencia humana ordinaria a partir de hacer cosas simples, como montar en bicicleta y cocinar una putxera con productos locales ${ }^{16}$.

\section{Referencias}

Augé, Marc. 1996. Los no lugares: Espacios del anonimato. Traducción, Margarita N. Mizraji. Barcelona: Gedisa

Blanco, Paloma, Jesús Carrillo et al., eds. 2001. Modos de hacer. Arte crítico, esfera pública y acción directa. A.F.R.I.K.A. Gruppe et al. Salamanca: Universidad de Salamanca

Díaz Viana, Luis. 2013. Narración y memoria: Anotaciones para una antropología de la catástrofe. Madrid: UNED

Hobsbawm, Eric \& Terence Ranger, eds. 2002. La invención de la tradición. Traducción castellana de Omar Rodríguez Estellar. Barcelona: Crítica

Lía Bang, Claudia. 2013. "El arte participativo en el espacio publico y la creación colectiva para la transformación social: Creatividad y arte”. Creatividad y Sociedad 20: 1-25 www. creatividadysociedad.com/articulos/20/2.\%20El\%20arte\%20participativo\%20en\%20el\%20 espacio\%20publico.pdf

Martí i Pérez, Josep. 1996. El folklorismo: Uso y abuso de la tradición. Barcelona: Rosel

\section{Notas}

1 Video anuncio de la Putxeramobil. Disponible en: https://vimeo.com/74750869. Acceso, 21 sept. 2016. 
2 'Poteo' quiere decir realizar un recorrido, normalmente en kuadrilla, por un barrio o pueblo con el hecho justificado de tomar un pote (vino, cerveza, etc.) en cada taberna que se visita. El bertso-poteo añade bertsos a este itinerario.

${ }^{3}$ Bertso-bazkari: recital de sobremesa. Actuaciones que se celebran en la sobremesa de comidas o cenas populares expresamente organizadas para ello.

${ }^{4}$ Las txosnas son una especie de tabernas que se montan en las calles durante la celebración de las fiestas en Euskal Herria, similares a las casetas en Andalucía. Estos establecimientos eventuales son organizados por los diferentes colectivos del lugar con el objetivo de contribuir a sus fiestas, a la vez que generan una ganancia para sus necesidades. Hay txosnas formadas por asambleas de jóvenes (gazte asambladas), por grupos de danzas, por asociaciones deportivas, otras de carácter político, ecológico, etc.

${ }^{5}$ Hay que puntualizar que poco a poco cada vez son más las personas que saben euskera, y los que se relacionan en un mundo euskaldun, gracias a muchas de las propuestas organizadas por la Bertso Eskola de Zalla, junto al respaldo del departamento de normativa lingüística (servicio de euskera) de la Mancomunidad de Municipios de las Encartaciones que promueve una gran cantidad de actividades, cursillos, campañas, etc., con el objetivo de difundir el uso del euskera en Enkarterri.

${ }^{6}$ Comprometidos con la promoción del euskera.

7 Traducción: Bueno, la putxera no será la mejor / para ver las alubias. / Que aquí el día va a ser en vano, / no se lo cree nadie. / Maquinista pon un trozo de madera / en estas ruedas, / aquí la mayoría de la madera / se necesita para llevar la bicicleta.

${ }^{8}$ Traducción: Y todavía ahí tenemos la putxera. / Desde el tren de la Robla / hasta la zona de Zalla. / ¡Ay! Señor maquinista, Fredi te pregunta: / ¿Cuándo se come aquí?

9 Traducción: Como hay un tiempo perfecto / damos gracias a Dios. / El hecho de llenar la tripa / cuánto placer nos crea a los euskaldunes / con una buenas alubias han llenado / a estos vagos bertsolaris / qué catalinas y qué piñones más buenos.

10 Traducción: Que el menú era Bueno / tengo que reconocer también / antes el cocinero / estaba fuera de este poteo. / Hoy al de nuestro grupo / lo teníamos con nosotros, / porque teníamos txirrin-dabas (bici-alubias). I Un fuerte aplauso merece / nuestro cocinero.

11 Traducción: Ciclista cocinero llenando nuestros platos. / Es increíble en Euskal Herria / el arte de la gastronomía. / Por casualidad en las alubias soy un experto / y es muy bueno el toque que le ha dado / el EPO (dopaje) a nuestras alubias.

12 Página web del proyecto de investigación Tecno_blandas impulsada por la cooperativa ColaBoraBora, dedicada al diseño y facilitación de entornos y procesos colaborativos centrados en las personas; y la artista Saioa Olmo, cuya práctica experimenta con cuestiones relativas a la psicología social a través de acciones grupales performativas. Este proyecto se lleva a cabo en el marco de la Unidad de Formación e Investigación (UFI) del Departamento de Arte y Tecnología de la Facultad de Bellas Artes de la UPV-EHU y del Grupo de Trabajo de Aprendizaje Expandido de Hirikilabs de Tabakalera. Disponible en: www. tecnologiasblandas.cc. Acceso, 20 de septiembre de 2016.

${ }^{13}$ Más información sobre este proyecto puede encontrarse en blog de la Txalamobil: http://txalamobil.blogspot.com.es. Acceso, 17 sept. 2016.

${ }^{14}$ Desde la filosofía de la Txalamobil, surgió la Txalabilbote, bajo los conceptos de preservar y crear. La Txalabilbote es un proyecto del Komando Trini (Edurne González Ibáñez y Txoni Manoli) y la Pandeira Audiovisual (Sabela Pernas Soto) creado a partir de la fusión entre un hidropedal y una txalaparta, con el objetivo de hacer un recorrido por la ría de Bilbao 
para interactuar con los transeúntes. El video de la botadura de la Txalabilbote desarrollado por Sabela Pernas Soto puede encontrarse en línea en: www.youtube.com/watch?v=zkOZFkIwBPo. Acceso, 17 sept. 2016.

${ }^{15}$ Proyecto Bicine, construcción de una pequeña pantalla de cine sobre una bicicleta. Puede consultarse en línea en: http://folklorenomada.com/section/198692-Bicine.html. Acceso, 17 sept. 2016.

${ }^{16}$ Video resumen de la Putxeramobil el Día de Gangas. Disponible en: www.youtube.com/watch?v=WU556zgBBRQ. Acceso, 21 sept 2016. 